\title{
Optimal portfolio and spending rules for endowment funds
}

\author{
Muhammad Kashif $^{1}$ (D) Francesco Menoncin ${ }^{1}$ (D) $\cdot$ Iqbal Owadally $^{2}$ OD \\ Published online: 18 November 2019 \\ (c) The Author(s) 2019
}

\begin{abstract}
We investigate the role of different spending rules in a dynamic asset allocation model for university endowment funds. In particular, we consider the fixed consumption-wealth ratio (CW) rule and the hybrid rule which smoothes spending over time. We derive the optimal portfolios under these two strategies and compare them with a theoretically optimal (Merton) strategy. We show that the optimal portfolio with habit is less risky compared to the optimal portfolio without habit. A calibrated numerical analysis on U.S. data shows, similarly, that the optimal portfolio under the hybrid strategy is less risky than the optimal portfolios under both the CW and the classical Merton strategies, in typical market conditions. Our numerical analysis also shows that spending under the hybrid strategy is less volatile than the other strategies. Thus, endowments following the hybrid spending rule use asset allocation to protect spending. However, in terms of the endowment's wealth, the hybrid strategy comparatively outperforms the conventional Merton and CW strategies when the market is highly volatile but under-performs them when there is strong stock market growth and low volatility. Overall, the hybrid strategy is effective in terms of stability of spending and intergenerational equity because, even if it allows short-term fluctuation in spending, it ensures greater stability in the long run.
\end{abstract}

Keywords Spending rules · Endowments $\cdot$ Bellman's equation $\cdot$ Asset allocation

JEL Classification G23 · C61 - G11

Iqbal Owadally

m.i.owadally@ city.ac.uk

Muhammad Kashif

muhammadkashif0027@gmail.com

Francesco Menoncin

francesco.menoncin@unibs.it

1 Department of Economics and Management, University of Brescia, Via S. Faustino, 74/B, 25122 Brescia, Italy

2 Cass Business School, City, University of London, 106 Bunhill Row, London EC1Y 8TZ, UK 


\section{Introduction}

University endowment funds rank among the largest institutional investors. In the National Association of College and University Business Officers (NACUBO) estimated that its member organizations in the U.S. held $\$ 515$ billion in endowment assets (NACUBO 2016). These funds pool donations from supporters and alumni, and are invested to support the current and future expenses of universities. Optimal investment and spending strategies for university endowments have been the subject of academic and professional scrutiny since Tobin (1974) and Merton (1993). Universities and colleges set up endowment funds to ensure a reasonably smooth earnings and consequent smooth stream of spending for current and future beneficiaries to preserve equity among generations [also called intergenerational equity, see Tobin (1974)]. To achieve this objective, many endowment funds set pre-defined spending rules. Hansmann (1990) addresses the reason why endowments would need such rules and concludes that endowments are established to fulfill some basic purposes which include: (i) ensure the support of the parent institution in its ongoing mission, (ii) protect its reputation and intellectual freedom, and (iii) hedge against financial shocks. In general, following a pre-defined spending rule is not optimal. As a result, endowment funds may invest too much in riskless assets which generally have lower returns.

Kaufman and Woglom (2005) discuss the role of spending rules in endowment funds. They analyze the spending rules based on the inflation method, banded inflation, and hybrid method, using Monte Carlo simulations in a scenario of volatile and uncertain asset returns. Ennis and Williamson (1976) present different spending rules adopted by endowment funds along with their historical spending patterns. Sedlacek and Jarvis (2010) provide an analysis of endowments' current practices and spending policies with their relative merit and demerits.

In this paper, we examine investment strategies under two spending rules in particular: (i) the fixed consumption-wealth ratio $(\mathrm{CW})$ rule, and (ii) the hybrid rule, a simplified form of the weighted average method commonly used by large endowment funds like Yale and Stanford (Cejnek et al. 2014). Under the CW rule, annual spending is a percentage of the market value of the fund, whereas the hybrid rule calculates spending as a weighted sum of the spending in the previous period and spending according to the fixed consumption-wealth ratio rule. To analyze the effect of the spending rules on risk-taking, we derive the optimal portfolio under the abovementioned spending rules for hyperbolic absolute risk aversion utility function and compare them with the classical Merton optimal investment and consumption. We also consider a detailed numerical example to investigate the effectiveness of the spending rules.

An endowment fund may exhibit intertemporal preferences where past spending generates a desire (or need) to maintain the same spending level into the future. Such a behavior can be modeled through habit formation or habit persistence, which is a more general case of subsistence level consumption. Actually, the habit can be interpreted as a time-varying subsistence consumption. In the Merton case, we consider a general form of utility function including both the cases of habit formation and subsistence level. Under the spending rules mechanism, endowments following the hybrid spending rule protect spending by investing in a less risky portfolio than Merton's. Similarly, investment in the risky asset with habit is less than the investment without habit. Thus, this strategy is similar to portfolio insurance where the fund invests in safe assets to maintain the value needed for having smooth payouts over time. 
The dynamic asset allocation and consumption studied in this paper is built upon the seminal work by Samuelson (1969) and Merton (1969), who present an optimal strategy for a market with constant investment opportunities with additive time-separable utility function. This preliminary work was later extended by Merton (1971), to a more general utility function which includes the income generated by non-capital gains sources.

This paper is related to a number of other works in the literature. Merton (1993) applies continuous time framework to the endowment fund's problem and derives optimal expenditures and asset allocation strategy that include non-endowed funds as a part of the total university's wealth. He concludes that endowment funds prefer a safer portfolio in the presence of non-financial income risk. Dybvig (1999) suggests that an endowment fund spending can be sustainable if some TIPS (Treasury Inflation Protection Securities) are held in the portfolio. Blume (2010) discusses the spending rules for endowments in volatile markets and illustrates the relation between spending rules and investment strategy under the assumption that asset classes with greater expected long-run returns display greater short-term volatility. He proposes that an endowment should choose a portfolio with a lower long run return when its value falls. BajeuxBesnainou and Ogunc (2006) address the asset allocation problem of an endowment fund by including, in the objective function, a minimum spending amount up-rated with inflation and obtain an explicit formula for optimal spending and portfolio allocation rules. Constantinides (1990) applies the habit formation to the equity puzzle problem and shows that the high equity premium with low risk aversion can be explained by the presence of habit formation. Munk (2008) studies the optimal strategies with general asset price dynamics under two special cases of time varying investment opportunities: stochastic interest rate, and mean-reverting stock returns. He shows that, in order to finance the habit, investment in bonds and cash is more effective than in stocks.

The rest of the paper is structured in the following way. Section 2 introduces the general framework for market dynamics, preferences of endowment fund, and endowment fund investment strategies, while Sect. 3 focuses on the results of optimal investment and spending under different strategies. Section 4 presents a numerical application of the previous results, and, finally, Sect. 5 concludes. Some technical derivations are left to "Appendix A".

\section{General framework}

Endowment funds usually invest in a variety of assets. For the purpose of tractability, we consider two assets listed in a complete and arbitrage-free, continuously open financial market:

- A riskless asset $G(t)$ which evolves according to

$$
\frac{d G(t)}{G(t)}=r d t
$$

where $G(0)=1$, and which is the numéraire and $r$ is a constant nominal interest rate;

- A risky asset $S(t)$ having the price dynamics given by 


$$
\frac{d S(t)}{S(t)}=\mu d t+\sigma d W(t)
$$

The endowment fund holds $\theta(t)$ units of the risky asset $S(t)$, and $\theta_{G}(t)$ units of risk-less assets $G(t)$. Thus, at any instant in time $t$, the investor's wealth $R(t)$ is given by

$$
R(t)=\theta(t) S(t)+\theta_{G}(t) G(t) .
$$

The differential of wealth can be written as

$$
d R(t)=(R(t) r+\theta(t) S(t)(\mu-r)-c(t)) d t+\theta(t) S(t) \sigma d W(t),
$$

where $c(t)$ is the instantaneous spending (or consumption) rate per unit time.

\subsection{Endowment fund spending and investment strategies}

In practice, endowment funds follow different spending rules to preserve endowment's wealth: see Sedlacek and Jarvis (2010) and Cejnek et al. (2014). We consider the fixed consumptionwealth ratio $(\mathrm{CW})$ rule and the hybrid rule for our optimal investment problem. We consider three strategies, a standard Merton strategy, a strategy with the CW rule and a strategy with the hybrid spending rule.

\subsubsection{Merton strategy}

In the Merton strategy, both investment and spending are decision variables, and we also generalize to include habit formation.

\subsubsection{Fixed consumption-wealth ratio (CW) strategy}

Investment is the only decision variable. Spending $c(t)$ in year $t$ equals a predetermined proportion $y$ of the endowment fund's wealth $R(t)$ :

$$
c(t)=y R(t)
$$

where $y$ is a constant and represents a fixed consumption-wealth ratio.

\subsubsection{Hybrid strategy}

Investment is the only decision variable. Spending is a weighted sum of the spending in the previous period and the spending under the fixed consumption-wealth ratio rule. In a discretetime setting, spending $c(t)$ is given by

$$
c(t+1)=\omega c(t)+(1-\omega) y R(t+1),
$$

where $\omega$ is the weight. This spending rule is a simplified version of the Yale/Stanford rule (Cejnek et al. 2014), which takes a weighted average of the inflation-adjusted spending of the previous period and a pre-specified percentage of a 3-year moving average of quarterly market values of the endowment fund.

The process in Eq. (5) is stationary if we assume $|\omega|<1$ and if $R(t)$ is also stationary. If $\omega$ is equal to zero, then (5) coincides with the fixed consumption-wealth ratio (CW) rule (4). We can rewrite Eq. (5) in continuous time as 


$$
d c(t)=(1-\omega)(y R(t)-c(t)) d t+(1-\omega) y d R(t) .
$$

\subsection{General settings}

We can write the endowment fund's utility functions for instantaneous outflow or spending $c(t)$ and final wealth $R(T)$ as

$$
U_{c}(c(t), h(t))=\frac{(c(t)-h(t))^{1-\delta}}{1-\delta}, \quad U_{R}(R(T))=\frac{\left(R(T)-R_{m}\right)^{1-\delta}}{1-\delta},
$$

where $\delta>1$ and $h(t)$ depends on the context: it is either a function representing the habit formation or a constant representing the subsistence level of spending. $R_{m}$ is a constant can be interpreted as the minimum subsistence level of wealth. Given the utility function (7), it is always optimal to have outflows higher than the threshold $h(t)$. In fact, when $c(t)=h(t)$, the marginal utility of the outflow tends towards infinity and, accordingly, it is sufficient to increase spending by an infinitesimal amount in order to have an infinite increase in the utility level.

The corresponding Arrow-Pratt Absolute Risk Aversion (ARA) indices of (7) are given by

$$
\begin{gathered}
-\frac{\partial^{2} U_{c}(c(t), h(t)) / \partial c(t)^{2}}{\partial U_{c}(c(t), h(t)) / \partial c(t)}=\frac{\delta}{c(t)-h(t)}, \\
-\frac{\partial^{2} U_{R}(R(T)) / \partial R(t)^{2}}{\partial U_{R}(R(T)) / \partial R(t)}=\frac{\delta}{R(T)-R_{m}},
\end{gathered}
$$

respectively, which implies that the higher $\delta$ is, the higher the risk aversion. Moreover, the higher $h(t)$ is, the higher is the risk aversion. This shows that having a higher level of minimum outflows means that it is necessary to invest larger amounts of wealth in the riskless asset in order to guarantee the outflows. If $h(t)$ and $R_{m}$ in (7) equals a positive constant, then the respective utility function belongs to the Hyperbolic Absolute Risk Aversion (HARA) family. However, if $h(t)$ and $R_{m}$ are equal to zero, then their respective utility function belongs to the Constant Relative Risk Aversion (CRRA) family.

According to Fraser and Jennings (2010), an endowment fund must define its investment policy statement by identifying the investment beliefs, specific investment objectives, rebalancing policy and performance benchmark which are evaluated periodically. Since an endowment fund must report its performance for each accounting period, it is reasonable to consider the optimization problem for a finite time interval $[t, T]$. If we assume the objective of an endowment fund is to maximize the sum of expected utility of spending and the expected utility of the final wealth, then the optimization problem can be stated as

$$
\max _{\pi(s)} \mathbb{E}_{t}\left[\int_{t}^{T} U_{c}(c(s), h(s)) e^{-\rho(s-t)} d s+U_{R}(R(T)) e^{-\rho(T-t)}\right],
$$

where the endowment fund chooses the decision variables $\pi(s)$, which may include spending and investment depending on the strategy considered and $\rho$ is a constant subjective discount rate. 


\section{The optimal solutions}

\subsection{Merton strategy}

Investment and spending are both decision variables, i.e. $\pi(t) \equiv\{c(t), \theta(t)\}$. We can define the value function using (8) as

$$
J(t, R(t)) \equiv \max _{c(s), \theta(s)} \mathbb{E}_{t}\left[\int_{t}^{T} \phi_{c} \frac{(c(s)-h(s))^{1-\delta}}{1-\delta} e^{-\rho(s-t)} d s+\phi_{R} \frac{\left(R(T)-R_{m}\right)^{1-\delta}}{1-\delta} e^{-\rho(T-t)}\right],
$$

where $\phi_{c}$ and $\phi_{R}$ are constants which measure the subjective relevance of the utility obtained from intertemporal spending and final wealth, respectively, and $h(t)$ is given by

$$
h(t)= \begin{cases}h_{0} e^{-\int_{0}^{t} \beta(u) d u}+\int_{0}^{t} \alpha(s) c(s) e^{-\int_{s}^{t} \beta(u) d u} d s, & \text { habit formation, } \\ h, & \text { subsistence level } \\ 0, & \text { classical problem }\end{cases}
$$

Here, $h_{0}$ is the initial minimum amount of outflow, $\alpha(t)$ is the weighting function providing the relative importance to the past outflow in computing the threshold $h(t)$, while $\beta(t)$ is a discount rate. In the habit formation case, $h(t)$ can be rewritten in continuous time as

$$
d h(t)=(\alpha(t) c(t)-\beta(t) h(t)) d t .
$$

Proposition 1 Given the state variable wealth $R(t)$ described in (3), the optimal spending and portfolio which solve problem (9) are

- In the case with habit formation:

$$
\begin{gathered}
c(t)^{*}=h(t)+\phi_{c}^{\frac{1}{\delta}} \frac{(R(t)-h(t) B(t))(1+B(t) \alpha(t))^{-\frac{1}{\delta}}}{A(t)}, \\
\theta(t)^{*}=\frac{\mu-r}{S(t) \sigma^{2}} \frac{R(t)-h(t) B(t)}{\delta},
\end{gathered}
$$

where

$$
\begin{aligned}
A(t)= & \phi_{R}^{\frac{1}{\delta}} e^{-\left(\frac{\delta-1}{\delta} r+\frac{\rho}{\delta}+\frac{(\delta-1)(\mu-r)^{2}}{2 \delta^{2} \sigma^{2}}\right)(T-t)} \\
& +\int_{t}^{T} \phi_{c}^{\frac{1}{\delta}}(1+B(s) \alpha(s))^{\frac{\delta-1}{\delta}} e^{-\left(\frac{\delta-1}{\delta} r+\frac{\rho}{\delta}+\frac{(\delta-1)(\mu-r)^{2}}{2 \delta^{2} \sigma^{2}}\right)(s-t)} d s, \\
B(t)= & R_{m} e^{-\int_{t}^{T}(-\alpha(u)+\beta(u)+r) d u}+\int_{t}^{T} e^{-\int_{t}^{s}(-\alpha(u)+\beta(u)+r) d u} d s .
\end{aligned}
$$

- In the case of a subsistence level: 


$$
c(t)^{*}=h+\phi_{c}^{\frac{1}{\delta}} \frac{R(t)-h B(t)}{A(t)}, \quad \theta(t)^{*}=\frac{\mu-r}{S(t) \sigma^{2}} \frac{R(t)-h B(t)}{\delta} .
$$

- In the classical case:

$$
c(t)^{*}=\phi_{c}^{\frac{1}{\delta}} \frac{R(t)}{A(t)}, \quad \theta(t)^{*}=\frac{\mu-r}{S(t) \sigma^{2}} \frac{R(t)}{\delta},
$$

where for both the subsistence level case and the classical case we have

$$
\begin{aligned}
& A(t)=\phi_{R}^{\frac{1}{\delta}} e^{-\left(\frac{\delta-1}{\delta} r+\frac{\rho}{\delta}+\frac{(\delta-1)(\mu-r)^{2}}{2 \delta^{2} \sigma^{2}}\right)(T-t)}+\phi_{c}^{\frac{1}{\delta}} \frac{1-e^{-\left(\frac{\delta-1}{\delta} r+\frac{\rho}{\delta}+\frac{(\delta-1)(\mu-r)^{2}}{2 \delta^{2} \sigma^{2}}\right)(T-t)}}{\frac{\delta-1}{\delta} r+\frac{\rho}{\delta}+\frac{(\delta-1)(\mu-r)^{2}}{2 \delta^{2} \sigma^{2}}}, \\
& B(t)=R_{m} e^{-r(T-t)}+\frac{1-e^{-r(T-t)}}{r} .
\end{aligned}
$$

\section{Proof See "Appendix A".}

In the case of habit formation, the function $A(t)$ in the optimal solutions is the weighted sum of two discount factors: (i) the discount factor for the final date $T$ multiplied by $\phi_{R}^{1 / \delta}$, and (ii) a kind of intertemporal discount factor for the intertemporal utility, multiplied by $\phi_{c}^{1 / \delta}$. The function $B(t)$ in the optimal solutions, is the sum of two terms, the subsistence wealth $R_{m}$ appropriately discounted from time $T$ and a sum of discount factors. We can see that habit formation has an effect on the optimal portfolio of the risky asset, as it changes the allocation due to the reason that the riskless asset (Treasury bill) is comparatively a better investment than the risky asset (stock) to ensure that the future spending will not decline below the habit level.

In the subsistence level and classical case, the function $A(t)$ in the optimal solutions is the weighted sum of two discount factors: (i) the discount factor for the final date $T$ multiplied by $\phi_{R}^{1 / \delta}$, and (ii) a term multiplied by $\phi_{c}^{1 / \delta}$. The function $B(t)$ in the optimal solutions, is the sum of two terms, the subsistence wealth $R_{m}$ appropriately discounted from time $T$ and a term which is positive and decreasing over time. The optimal spending in the subsistence level case may be shown to be consistent with the result obtained by BajeuxBesnainou and Ogunc (2006).

We consider the optimal spending and investment in the habit formation case defined in (12) and (13), respectively, in detail below.

Assumption 1 We assume that the parameters $\alpha$ and $\beta$ are constants over time and additionally $r-\alpha+\beta>0$.

Corollary 1 Under Assumption 1, (i) The functions A(t) and B(t) can be written as 


$$
\begin{aligned}
A(t)= & \phi_{R}^{\frac{1}{\delta}} e^{-\left(\frac{\delta-1}{\delta} r+\frac{\rho}{\delta}+\frac{(\delta-1)(\mu-r)^{2}}{2 \delta^{2} \sigma^{2}}\right)(T-t)} \\
& +\int_{t}^{T} \phi_{c}^{\frac{1}{\delta}}(1+B(s) \alpha)^{\frac{\delta-1}{\delta}} e^{-\left(\frac{\delta-1}{\delta} r+\frac{\rho}{\delta}+\frac{(\delta-1)(\mu-r)^{2}}{2 \delta^{2} \sigma^{2}}\right)(s-t)} d s, \\
B(t)= & R_{m} e^{-(r-\alpha+\beta)(T-t)}+\int_{t}^{T} e^{-(r-\alpha+\beta)(s-t)} d s=R_{m} e^{-(r-\alpha+\beta)(T-t)} \\
& +\frac{1-e^{-(r-\alpha+\beta)(T-t)}}{r-\alpha+\beta}
\end{aligned}
$$

and finally, if we substitute the value of $B(t)$ into $A(t)$, we get

$$
\begin{aligned}
A(t)= & \phi_{R}^{\frac{1}{\delta}} e^{-\left(\frac{\delta-1}{\delta} r+\frac{\rho}{\delta}+\frac{(\delta-1)(\mu-r)^{2}}{2 \delta^{2} \sigma^{2}}\right)(T-t)} \\
& +\int_{t}^{T} \phi_{c}^{\frac{1}{\delta}}\left(1+R_{m} e^{-(r-\alpha+\beta)(T-s)}\right. \\
& \left.+\frac{1-e^{-(r-\alpha+\beta)(T-s)}}{r-\alpha+\beta} \alpha\right)^{\frac{\delta-1}{\delta}} e^{-\left(\frac{\delta-1}{\delta} r+\frac{\rho}{\delta}+\frac{(\delta-1)(\mu-r)^{2}}{2 \delta^{2} \sigma^{2}}\right)(s-t)} d s .
\end{aligned}
$$

(ii) The optimal spending and portfolio are

$$
\begin{gathered}
c(t)^{*}=h(t)+\phi_{c}^{\frac{1}{\delta}} \frac{(R(t)-h(t) B(t))(1+B(t) \alpha)^{-\frac{1}{\delta}}}{A(t)}, \\
\theta(t)^{*}=\frac{\mu-r}{S(t) \sigma^{2}} \frac{R(t)-h(t) B(t)}{\delta},
\end{gathered}
$$

where $B(t)$ and $A(t)$ are given by (16) and (17).

The term

$$
\frac{1-e^{-(r-\alpha+\beta)(T-s)}}{r-\alpha+\beta}
$$

is positive and decreasing over time as $r-\alpha+\beta>0$.

(iii) The optimal portfolio with habit is less risky than the optimal portfolio without habit:

$$
S(t) \theta(t)^{*}<\left.S(t) \theta(t)^{*}\right|_{h(t)=0} .
$$

Proof The optimal amount invested in the stock $S(t) \theta(t)^{*}$ is given by

$$
\frac{\mu-r}{S(t) \sigma^{2}} \frac{R(t)-h(t) B(t)}{\delta},
$$

Since by construction $h(t) \geq 0$ and $B(t) \geq 0$, thus

$$
\frac{\mu-r}{S(t) \sigma^{2}} \frac{R(t)-h(t) B(t)}{\delta}<\frac{\mu-r}{S(t) \sigma^{2}} \frac{R(t)}{\delta} .
$$


Endowment funds are long-lived, typically infinitely-lived, investors. We take into account long-range investment and budget planning of university endowments by considering the case when $T \rightarrow \infty$.

Corollary 2 Under Assumption 1 and with $T \rightarrow \infty$, the optimal spending and portfolio are

$$
\begin{aligned}
& c(t)^{*}=h(t)+\phi_{c}^{\frac{1}{\delta}} \frac{\left(R(t)-h(t) \frac{1}{r-\alpha+\beta}\right)\left(1+\frac{\alpha}{r-\alpha+\beta}\right)^{-\frac{1}{\delta}}}{A(t)}, \\
& \theta(t)^{*}=\left(R(t)-\frac{h(t)}{r-\alpha+\beta}\right) \frac{\mu-r}{S(t) \sigma^{2} \delta},
\end{aligned}
$$

where

$$
A(t)=\frac{\phi_{c}^{\frac{1}{\delta}}\left(1+\frac{\alpha}{r-\alpha+\beta}\right)^{\frac{\delta-1}{\delta}}}{\left(\frac{\delta-1}{\delta} r+\frac{\rho}{\delta}+\frac{(\delta-1)(\mu-r)^{2}}{2 \delta^{2} \sigma^{2}}\right)} .
$$

In this case the dynamics of optimal wealth and habit are

$$
\begin{aligned}
d R(t)= & \left(R(t) r+\left(R(t)-\frac{h(t)}{r-\alpha+\beta}\right) \frac{(\mu-r)^{2}}{\sigma^{2} \delta}-h(t)\right. \\
& \left.-\phi_{c}^{\frac{1}{\delta}} \frac{\left.\left(R(t)-h(t) \frac{1}{r-\alpha+\beta}\right)\left(1+\frac{\alpha}{r-\alpha+\beta}\right)^{-\frac{1}{\delta}}\right) d t}{A(t)}\right) \\
& +\left(R(t)-\frac{h(t)}{r-\alpha+\beta}\right) \frac{\mu-r}{\sigma \delta} d W(t),
\end{aligned}
$$

and

$$
d h(t)=(\beta-\alpha)\left(\frac{\alpha}{\beta-\alpha} \phi_{c}^{\frac{1}{\delta}} \frac{\left(R(t)-h(t) \frac{1}{r-\alpha+\beta}\right)\left(1+\frac{\alpha}{r-\alpha+\beta}\right)^{-\frac{1}{\delta}}}{A(t)}-h(t)\right) d t
$$

where $A(t)$ is given in (20) and we can see that habit $h(t)$ is a mean reverting process if $\beta-\alpha>0$ and is instead exploding if $\beta-\alpha<0$.

\subsection{CW strategy}

In this case, investment $\theta(t)$ is the only decision variable, i.e., $\pi(t) \equiv \theta(t)$, and spending $c(t)$ is given by

$$
c(t)=y R(t)
$$

therefore we can put $\phi_{c}=0$ and $\phi_{R}=1$ in (9), whereupon the value function can be defined as 


$$
J(t, R(t)) \equiv \max _{\theta(t)} \mathbb{E}_{t}\left[\frac{\left(R(T)-R_{m}\right)^{1-\delta}}{1-\delta} e^{-\rho(T-t)}\right]
$$

Proposition 2 Given the state variable $R(t)$ and $c(t)$ described in (3) and (21), respectively, the optimal portfolio which solve problem (22) is

$$
\theta(t)^{*}=\frac{\mu-r}{S(t) \sigma^{2}} \frac{R(t)-B(t)}{\delta}
$$

where

$$
B(t)=R_{m} e^{-(r-y)(T-t)},
$$

and $y$ is the constant defined in (21).

Proof See "Appendix A".

The function $B(t)$ in Proposition 2 above is the subsistence wealth $R_{m}$ appropriately discounted. An elegant and illuminating solution to the infinite planning horizon problem may be obtained in the case that $r>y$ : see Corollary 3 below. (The restriction that $r>y$ is not required in Proposition 2, and is not made in the numerical analysis of Sect. 4.)

Corollary 3 Under Assumption 1, with $T \rightarrow \infty$ and $r>y$, the optimal portfolio (23) becomes

$$
\theta(t)^{*}=\frac{\mu-r}{S(t) \sigma^{2}} \frac{R(t)}{\delta}
$$

In this case the dynamics of optimal wealth is

$$
\frac{d R(t)}{R(t)}=\left(r+\frac{1}{\delta} \frac{(\mu-r)^{2}}{\sigma^{2}}-y\right) d t+\frac{1}{\delta} \frac{\mu-r}{\sigma} d W(t)
$$

\subsection{Hybrid strategy}

As in the CW strategy, investment is the only decision variable, while spending evolves according to the weighted average spending rule (6) in which we substitute (3) to get

$$
\begin{aligned}
& d c(t)=(1-\omega)(y R(t)-c(t)) d t+(1-\omega) y(R(t) r+\theta(t) S(t)(\mu-r)-c(t)) d t \\
& +(1-\omega) y \theta(t) S(t) \sigma d W(t),
\end{aligned}
$$

which can be rewritten as

$$
\begin{aligned}
d c(t) & =(1-\omega)(1+y)\left(\frac{y R(t)(1+r)+y \theta(t) S(t)(\mu-r)}{(1+y)}-c(t)\right) d t \\
+ & (1-\omega) y \theta(t) S(t) \sigma d W(t) .
\end{aligned}
$$

If we set $a \equiv(1-\omega) y$ then we can write 


$$
\begin{aligned}
& d c(t)=(1-\omega)(1+y)\left(\frac{y R(t)(1+r)+y \theta(t) S(t)(\mu-r)}{1+y}-c(t)\right) d t \\
& +a \theta(t) S(t) \sigma d W(t),
\end{aligned}
$$

where we see that the spending is a mean reverting process, whose strength of mean reversion is $(1-\omega)(1+y)$. The spending reverts towards

$$
\frac{y R(t)(1+r)+y \theta(t) S(t)(\mu-r)}{1+y},
$$

which depends on the portfolio choice. The higher the value of $\omega$, the more slowly $c(t)$ converges towards its long-term level and vice versa.

As the spending is given by (24), we include it as an additional state variable and put $\phi_{c}=0$ and $\phi_{R}=1$ in (9), hence the value function can be defined as

$$
J(t, R(t), c(t)) \equiv \max _{\theta(t)} \mathbb{E}_{t}\left[\frac{\left(R(T)-R_{m}\right)^{1-\delta}}{1-\delta} e^{-\rho(T-t)}\right] .
$$

Proposition 3 Given the state variables $R(t)$ and $c(t)$ as in (3) and (24) the optimal portfolio which solve problem (25) is

$$
\theta(t)^{*}=\frac{\mu-r}{S(t) \sigma^{2}} \frac{R(t)-B(t, c(t))}{\delta\left(1-\eta^{*} a\right)}
$$

where

$$
B(t, c(t))=\eta^{*} c(t)+R_{m} e^{-\left(r-\eta^{*} a(1+r)\right)(T-t)},
$$

and $\eta^{*}$ takes one of the following values

$$
\eta=\frac{(1+r+a-\omega) \pm \sqrt{(1+r+a-\omega)^{2}-4 a(1+r)}}{2 a(1+r)},
$$

such that $-\infty<\eta^{*}<\frac{1}{a}$ and $a=y(1-\omega)<1$.

Proof See "Appendix A".

Corollary 4 Under Assumption 1 , with $T \rightarrow \infty$ and if $r>\eta^{*} a(1+r)$, the optimal portfolio (26) becomes

$$
\theta(t)^{*}=\frac{\mu-r}{S(t) \sigma^{2}} \frac{R(t)-\eta^{*} c(t)}{\delta\left(1-\eta^{*} a\right)} .
$$

In this case the dynamics of optimal wealth and optimal spending are 
Table 1 Parameters calibrated on the S\&P 500 and U.S. 3-Month Treasury Bill time series between (i) January 2nd, 1997 and December 29th, 2006 (1997-2006), (ii) January 3rd, 2007 and December 30th, 2011 (2007-2011), and (iii) January 3rd, 2012 and December 30th, 2016 (2012-2016). Other assumptions include $R_{0}=100$, $T=10, R_{m}=0$ or 90 and $\delta=2$

\begin{tabular}{llll}
\hline Parameters & $1997-2006$ & $2007-2011$ & $2012-2016$ \\
\hline$\mu$ & 0.0816 & 0.0117 & 0.1198 \\
$\sigma$ & 0.1816 & 0.2659 & 0.1279 \\
$r, \rho$ & 0.0356 & 0.0122 & 0.0011 \\
\hline
\end{tabular}

$$
\begin{aligned}
d R(t)= & \left(R(t) r+\left(\frac{(\mu-r)^{2}}{\sigma^{2}} \frac{R(t)-\eta^{*} c(t)}{\delta\left(1-\eta^{*} a\right)}\right)-c(t)\right) d t \\
& +\left(\frac{\mu-r}{\sigma} \frac{R(t)-\eta^{*} c(t)}{\delta\left(1-\eta^{*} a\right)}\right) d W(t), \\
d c(t)= & \left(a R(t)(1+r)+a\left(\frac{(\mu-r)^{2}}{\sigma^{2}} \frac{R(t)-B(t, c(t))}{\delta\left(1-\eta^{*} a\right)}\right)-(1-\omega)(1+y) c(t)\right) d t \\
& +a\left(\frac{\mu-r}{\sigma} \frac{R(t)-B(t, c(t))}{\delta\left(1-\eta^{*} a\right)}\right) d W(t) .
\end{aligned}
$$

\section{A numerical application}

To illustrate the results of the preceding section, a simplified market structure is modeled under Assumption 1. Additionally, in order to compare and contrast the three strategies, we assume that the relative importance to the past outflow $\alpha$ and the discount rate $\beta$ are equal to zero, and thus there is no habit $(h(t)=0)$. We have estimated the parameters related to the financial market and interest rate over three different time horizons: (i) January 2, 1997 to December 29, 2006 (1997-2006), (ii) January 3, 2007 to December 30, 2011 (2007-2011), and (iii) January 3, 2012 to December 30, 2016 (2012-2016). The parameters of the dynamics of the risky asset price $S(t)$, in equation (2), are estimated from the S\&P 500, and the value of constant interest rate $r$ is estimated as the average return of U.S. 3-Month Treasury Bill (on secondary market - daily data). We assume that the risk aversion parameter $\delta=2$ similar to the most common choice of risk aversion parameter value in the habit formation and life cycle literature (Munk 2008; Horneff et al. 2015; Gourinchas and Parker 2002). We set the subjective discount factor $\rho$ equal to the riskless interest rate $r$. The estimated parameters along with some assumptions about wealth and preferences are gathered in Table 1.

We recall the general objective function under the assumption $h(t)=0$ :

$$
J(t, R(t)) \equiv \max _{\pi(t)} \mathbb{E}_{t}\left[\int_{t}^{T} \phi_{c} \frac{(c(s))^{1-\delta}}{1-\delta} e^{-\rho(s-t)} d s+\phi_{R} \frac{\left(R(T)-R_{m}\right)^{1-\delta}}{1-\delta} e^{-\rho(T-t)}\right],
$$



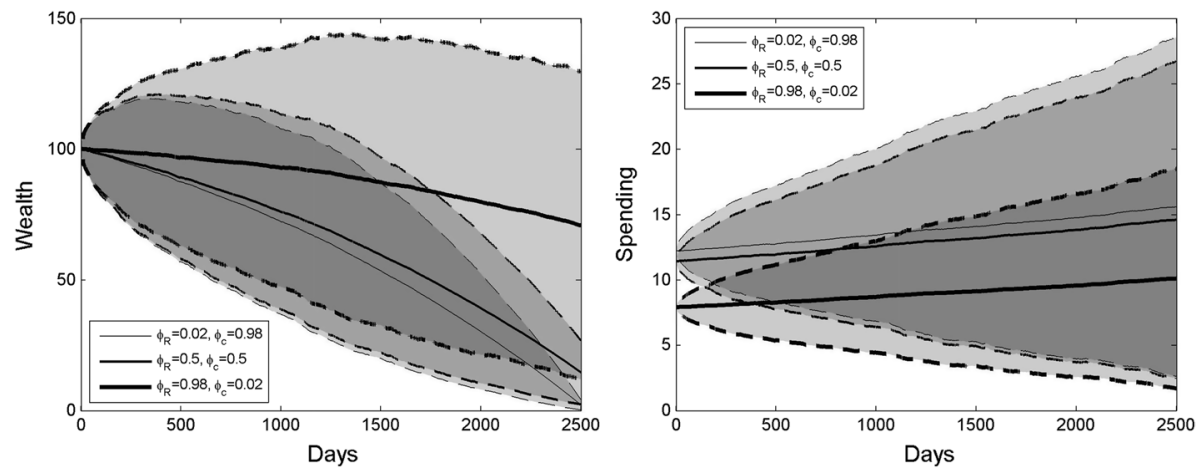

Fig. 1 Wealth and spending under Merton strategy with different values of the weights $\left(\phi_{c}\right.$ and $\left.\phi_{R}\right)$ in the objective function (27). Dashed lines show the confidence interval (i.e. mean plus and minus two standard deviations). Parameter values are estimated for the period 1997-2006 as stated in Table 1
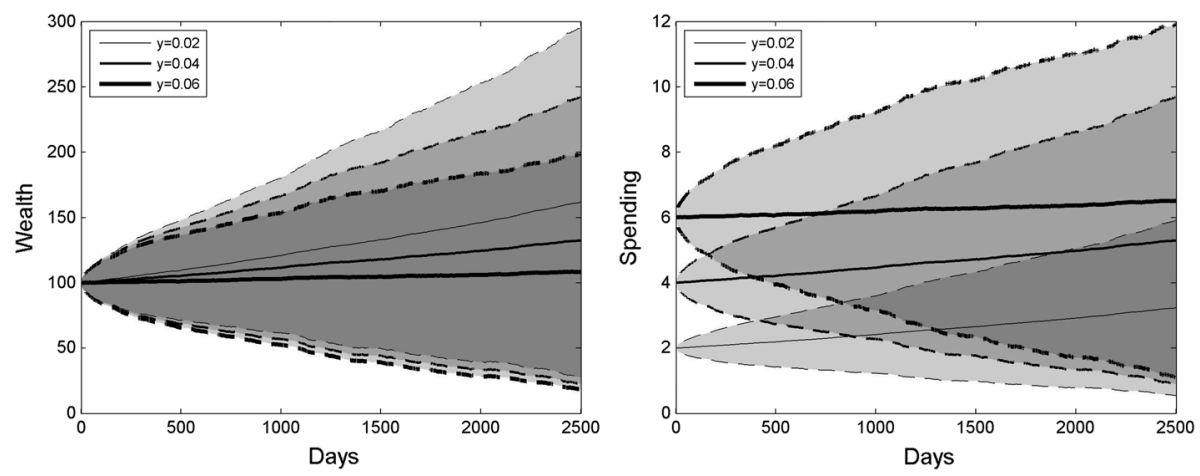

Fig. 2 Wealth and spending under CW strategy with different values of the consumption-wealth ratio $y$. Dashed lines show the confidence interval. Parameter values are estimated for the period 1997-2006 as stated in Table 1
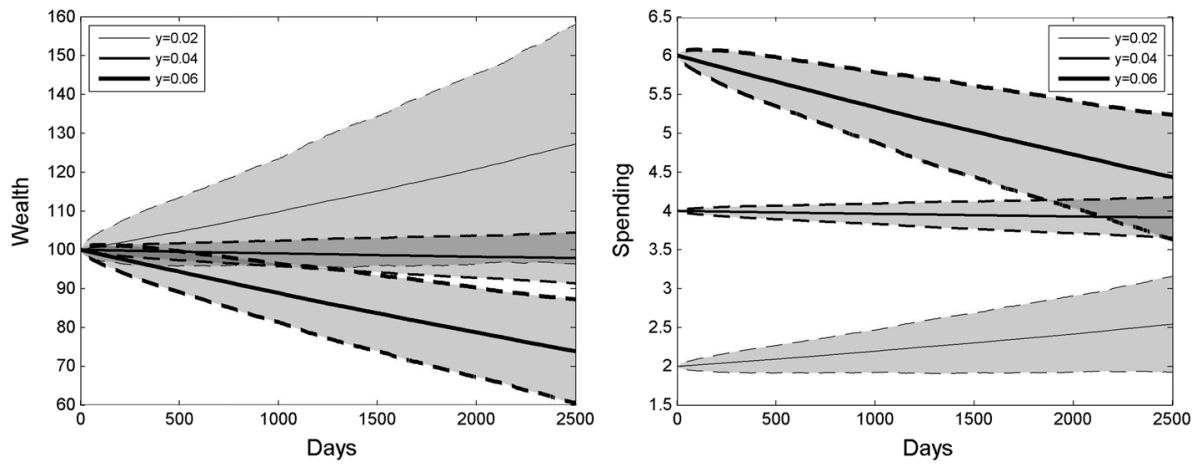

Fig. 3 Wealth and spending under CW strategy with different values of the consumption-wealth ratio $y$ when $R_{m}=90$. Dashed lines show the confidence interval. Parameter values are estimated for the period 1997-2006 as stated in Table 1 

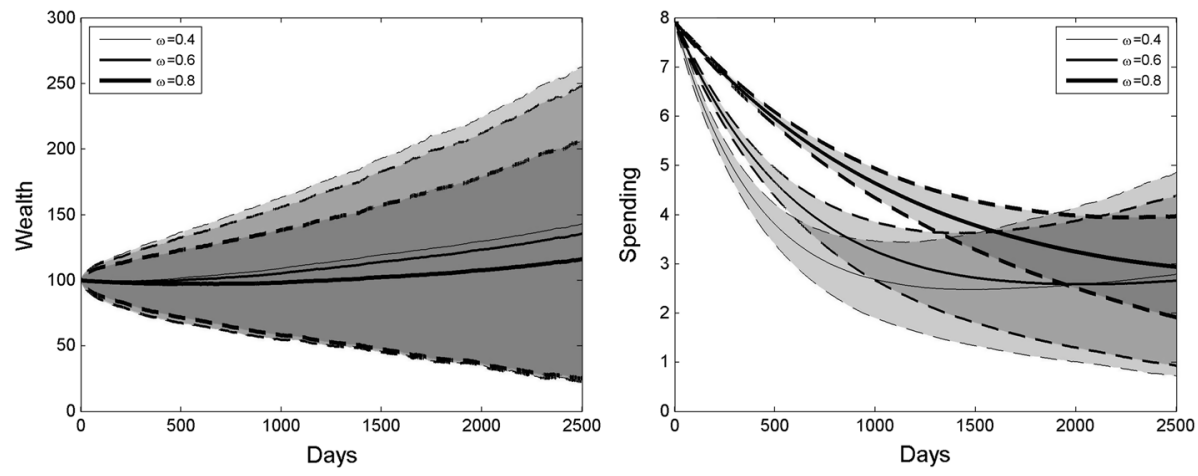

Fig. 4 Wealth and spending under the hybrid strategy with different values of the weight $\omega$. Dashed lines show the confidence interval. Parameter values are estimated for the period 1997-2006 as stated in Table 1
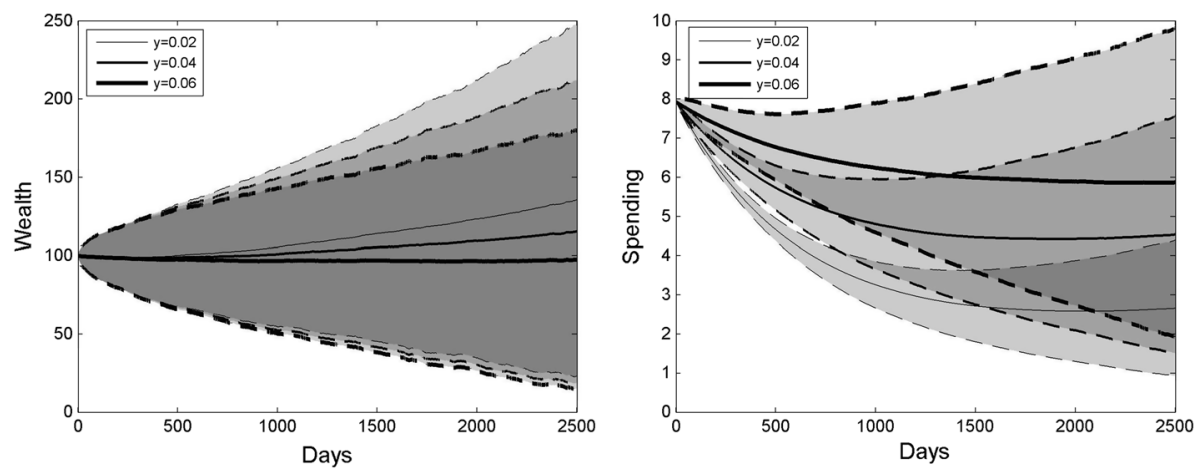

Fig. 5 Wealth and spending under the hybrid strategy with different values of $y$. Dashed lines show the confidence interval. Parameter values are estimated for the period 1997-2006 as stated in Table 1

In Figs. 1, 2, 3, 4, 5, the solid lines show the mean of 1000 simulations while the dashed lines with shaded areas show the confidence interval, i.e. mean plus and minus two standard deviations using the parameters estimated for the period 1997-2006. Figure 6 shows only the mean of 1000 simulations using the parameters estimated for the periods 1997-2006, 2007-2011 and 2012-2016.

Figure 1 illustrates the sensitivity of the wealth and spending to variation in weights assigned to the utility of terminal wealth and intertemporal spending in the objective function (27) under Merton strategy. The graph indicates that weights do affect the optimal portfolio and spending and a comparatively higher weight must be given to $\phi_{R}$ as compared with $\phi_{c}$ to maintain wealth above zero in the long run. However, higher $\phi_{c}$ leads to higher (and increasing) spending, increasing over time, as one would expect.

Figure 2 presents the impact of the consumption-wealth ratio $y$ on the paths of wealth and spending for the CW strategy, in the absence of subsistence wealth, i.e. when $R_{m}=0$. We can see that, when $y$ increases, the level of spending rises but the terminal wealth declines. For further analysis, we consider the case when the subsistence wealth $R_{m}$ is close to initial wealth $R_{0}$, i.e. $R_{m}=90$, the wealth and spending become less volatile for 


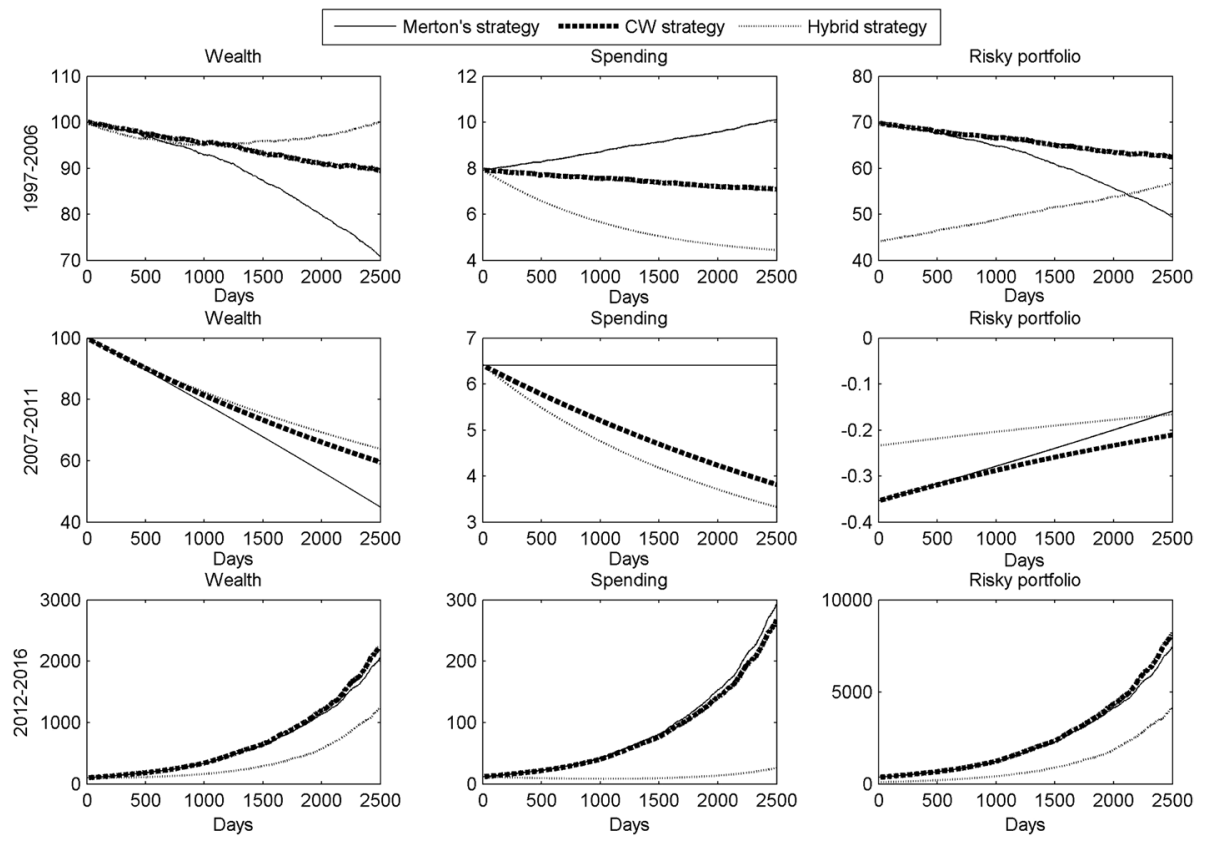

Fig. 6 Mean wealth, mean spending and mean amount invested in risky asset under the Merton strategy with $\phi_{R}=0.98$ and $\phi_{c}=0.02$ (continuous line), the CW strategy with $y=0.04$ (bold dashed line) and the hybrid strategy with $\omega=0.8$ and $y=0.04$ (dotted line). Parameter values are estimated for the periods 1997-2006, 2007-2011, and 2012-2016 as stated in Table 1

all values of $y$, as shown in the Fig. 3. As expected, we can see that a rise or decline in the wealth and spending in the long run depends on the ratio $y$.

Figure 4 shows the wealth and spending with different values of the weight $\omega$ for the hybrid strategy under the assumption that $R_{m}=0$. Interestingly, we can see from the graph that the weight $\omega$ must be chosen prudently: the higher the value of $\omega$, the more slowly $c(t)$ converges towards its long-term level. This is as expected since the more weight is given to past spending in the determination of future spending, the more slowly spending changes. Similar to the CW strategy, if $R_{m}$ is close to the initial wealth $R_{0}$, the paths of wealth and spending becomes less volatile. For this strategy, the initial spending must not be higher than a certain threshold, otherwise the wealth will decline to zero. In fact, endowment managers face a dilemma in deciding whether to have spending above a certain level to fund the current activities or to have spending lower than a threshold to achieve the long term objectives. Thus, in choosing the initial level of spending, the management must weigh the potential of greater spending levels in the long run against the need for current spending. The behavior of spending under the hybrid strategy appears to be more acceptable to endowment managers as: (i) it is less volatile in the long run compared to the Merton and CW strategies and (ii) it converges towards a long-term average level. In fact, an excessive volatility in spending is undesirable.

Figure 5 examines the sensitivity of the hybrid strategy to different values of constant $y$. We see that while $y$ increases, so does the volatility in wealth and spending which implies that $y$ must be chosen cautiously for the long term growth of wealth. The path of spending 
also depends on the value of $y$. If $y$ is higher than the optimal value, then the wealth will not grow in the long run and eventually, the spending will also decline to a much lower level. If $y$ is lower than the optimal level, then the wealth will grow in the long run but the spending will remain lower even in the long run, provided that the values of other parameters remain unchanged.

Figure 6 shows the mean wealth, spending and risky portfolio (all in monetary units) for the Merton, CW and hybrid strategies using the parameters estimated for three different periods: (i) 1997-2006, (ii) 2007-2011, and (iii) 2012-2016. The graphs in the first column show the dynamic behavior of wealth. We observe that:

- During the first period (1997-2006) under the hybrid strategy, average wealth initially declines and then recovers, while under the Merton strategy, it declines sharply.

- During the second, highly volatile, period (2007-2011), average wealth declines for all strategies but the magnitude of decline is less for the hybrid strategy compared to the other two strategies.

- During the third period (2012-2016) of low volatility and strong stock market growth, average wealth rises for all three strategies but it rises less strongly for the hybrid strategy.

The graphs in the second column of Fig. 6 show the dynamic behavior of spending. To effectively compare spending, we set the consumption-wealth ratio for the CW strategy and the initial spending for the hybrid strategy in order to match the initial optimal spending under the Merton strategy. We can see that:

- During the first period, average spending rises under the Merton strategy, while it declines under the hybrid strategy in the short run and converges towards a long-term level.

- During the second period, average spending remains constant under the Merton strategy, however it declines under the other two strategies.

- During the third period, average spending increases greatly for CW and Merton strategy, while it increases much more slowly for the hybrid strategy.

From the graphs in the third column of Fig. 6, which show the average amount held in the risky asset, we observe that

- During the less volatile periods (most of 1997-2006 and 2012-2016), the optimal portfolio under the hybrid strategy is on average less risky than under both the $\mathrm{CW}$ and Merton strategies.

- During the very volatile period of the financial crisis (most of 2007-2011), the hybrid strategy leads to a riskier portfolio on average compared to the other two strategies, but the risky asset is shorted on average under all three strategies.

- The hybrid strategy tends to adopt a more conservative stance, starting off with a less risky portfolio for example in 1997-2006 and 2012-2016, and gradually increases the holding of risky assets over time, in order to protect future spending and the corpus of the endowment fund. 


\section{Conclusion}

University endowment funds are long-term investors with specific objectives requiring particular investment and spending strategies. Endowment fund managers adopt different spending rules to effectively preserve the corpus of the fund and have a stable spending stream. We derived the optimal investment strategy under the fixed consumption-wealth ratio $(\mathrm{CW})$ rule and the hybrid spending rule. We compared these optimal portfolios and associated spending rules with the classical Merton optimal portfolio and consumption.

A numerical analysis using market data over three different periods in the last two decades showed that the hybrid strategy leads to a less risky portfolio than both the CW strategy and conventional Merton strategy, under typical market conditions. The hybrid strategy, which explicitly smoothes inter-temporal spending, thus operates similarly to an optimal Merton portfolio with habit formation. We also found in our numerical example that spending under the hybrid strategy appears to be less volatile than under the other two strategies. The unique characteristic of the hybrid rule is that it allows fluctuation in spending in the short run but stabilizes spending in the long run regardless of the initial allocation for spending. In terms of the endowment's wealth, the hybrid strategy performs better than the conventional Merton strategy and the CW strategy when the market is highly volatile but is outperformed in a low-volatility high-growth environment. Thus, an endowment fund must evaluate, review and modify its spending rule and investment policy periodically, depending on financial market conditions.

Future work will incorporate a stochastic interest rate environment for greater realism. Endowment fund expenditure across activities like research, teaching, estate upkeep, and administration can also be differentiated. The impact of spending and investment on these different areas may vary from one activity to another. We also intend to carry out empirical work to observe how our optimal solutions behave in stress scenarios, such as a liquidity crisis. $^{1}$

Open Access This article is distributed under the terms of the Creative Commons Attribution 4.0 International License (http://creativecommons.org/licenses/by/4.0/), which permits unrestricted use, distribution, and reproduction in any medium, provided you give appropriate credit to the original author(s) and the source, provide a link to the Creative Commons license, and indicate if changes were made.

\section{Appendix A1}

\section{Mathematical proofs}

For simplification of the notation, we will use the following definitions throughout this appendix:

$$
\frac{\partial A(t)}{\partial t} \equiv A_{t}, \quad \frac{\partial B(t, c(t))}{\partial t} \equiv B_{t}, \quad \frac{\partial B(t, c(t))}{\partial c} \equiv B_{c}, \quad \frac{\partial^{2} B(t, c(t))}{\partial^{2} c} \equiv B_{c c} .
$$

1 We are grateful to an anonymous reviewer for these suggestions. 


\section{Proof of proposition 1}

The value function or indirect utility function is given by

$$
\begin{aligned}
& J(t, R(t)) \equiv \max _{c(s), \theta(s)} \mathbb{E}_{t}\left[\int_{t}^{T} \phi_{c} \frac{(c(s)-h(s))^{1-\delta}}{1-\delta} e^{-\rho(s-t)} d s\right. \\
& \left.+\phi_{R} \frac{\left(R(T)-R_{m}\right)^{1-\delta}}{1-\delta} e^{-\rho(T-t)}\right] .
\end{aligned}
$$

We know that the above objective function must solve the following differential equation (so-called Hamilton-Jacobi-Bellman HJB equation):

$$
0=\max _{c(s), \theta(s)}\left\{\begin{array}{c}
\phi_{c} \frac{(c(s)-h(s))^{1-\delta}}{1-\delta}-\rho J+\frac{\partial J}{\partial t}+\frac{\partial J}{\partial h}(\alpha(t) c(t)-\beta(t) h(t))+\frac{\partial J}{\partial R}(R(t) r \\
+\theta(t) S(t)(\mu-r)-c(t))+\frac{1}{2} \frac{\partial^{2} J}{\partial R^{2}} \theta(t)^{2} S(t)^{2} \sigma^{2}
\end{array}\right\} .
$$

The HJB Eq. (28) in $J(t, R(t))$, needs a boundary condition so that the value function coincide with the final utility function at the time $T: J(T, R(T))=U(R(T))$.

We assume the following guess function

$$
J(t, R(t))=A(t)^{\delta} \frac{(R(t)-h(t) B(t))^{1-\delta}}{1-\delta},
$$

where $A(t)$ and $B(t)$ are the functions that must be determined to solve Eq. (28). Both functions must satisfy boundary conditions as follows:

$$
A(T)^{\delta}=\phi_{R} \Rightarrow A(T)=\phi_{R}^{\frac{1}{\delta}}, \quad B(T)=R_{m},
$$

and the first order conditions (FOCs) of (28) w.r.t. $\theta(t)$ and $c(t)$ are:

$$
\begin{gathered}
\theta(t)^{*}=-\frac{\mu-r}{S(t) \sigma^{2}} \frac{\frac{\partial J}{\partial R}}{\frac{\partial^{2} J}{\partial R^{2}}}, \\
\phi_{c}(c(s)-h(s))^{-\delta}=\frac{\partial J}{\partial R}-\frac{\partial J}{\partial h} \alpha(t) .
\end{gathered}
$$

By substituting the derivatives of the guess function into both the optimal spending (31) and the optimal portfolio (30), we obtain

$$
\begin{gathered}
c(t)^{*}=h(t)+\phi_{c}^{\frac{1}{\delta}} \frac{(R(t)-h(t) B(t))(1+B(t) \alpha(t))^{-\frac{1}{\delta}}}{A(t)}, \\
\theta(t)^{*}=\frac{\mu-r}{S(t) \sigma^{2}} \frac{R(t)-h(t) B(t)}{\delta} .
\end{gathered}
$$

Inserting the optimal spending (32), the optimal portfolio (33) and the partial derivatives of the guess function into Eq. (28), we have 


$$
\begin{gathered}
0=\frac{\delta}{1-\delta} \phi_{c}^{\frac{1}{\delta}}(1+B(t) \alpha(t))^{1-\frac{1}{\delta}}-\frac{\rho A(t)}{1-\delta}+\frac{\delta}{1-\delta} A_{t}-\frac{A(t) h(t) B_{t}}{R(t)-h(t) B(t)} \\
-\frac{A(t) B(t) \alpha(t) h(t)}{R(t)-h(t) B(t)}+\frac{A(t) B(t) \beta(t) h(t)}{R(t)-h(t) B(t)}+r A(t) \\
+\frac{A(t) h(t) B(t) r}{R(t)-h(t) B(t)}+A(t) \frac{(\mu-r)^{2}}{2 \sigma^{2} \delta}-\frac{A(t) h(t)}{R(t)-h(t) B(t)},
\end{gathered}
$$

which can be separated into two differential equations, one that consists of the terms containing $(R(t)-h(t) B(t))^{-1}$ and one without them and after few simplifications we have

$$
\left\{\begin{array}{l}
0=\phi_{c}^{\frac{1}{\delta}}(1+B(t) \alpha(t))^{\frac{\delta-1}{\delta}}+A_{t}+A(t)\left(\frac{1-\delta}{\delta} r-\frac{\rho}{\delta}+\frac{(1-\delta)(\mu-r)^{2}}{2 \delta^{2} \sigma^{2}}\right) \\
0=B_{t}+B(t)(\alpha(t)-\beta(t)-r)+1
\end{array}\right.
$$

The above ordinary differential equations, together with their corresponding boundary conditions, have the following unique solutions:

$$
\begin{aligned}
A(t) & =\phi_{R}^{\frac{1}{\delta}} e^{-\left(\frac{\delta-1}{\delta} r+\frac{\rho}{\delta}+\frac{(\delta-1)(\mu-r)^{2}}{2 \delta^{2} \sigma^{2}}\right)(T-t)} \\
& +\int_{t}^{T} \phi_{c}^{\frac{1}{\delta}}(1+B(s) \alpha(s))^{\frac{\delta-1}{\delta}} e^{-\left(\frac{\delta-1}{\delta} r+\frac{\rho}{\delta}+\frac{(\delta-1)(\mu-r)^{2}}{2 \delta^{2} \sigma^{2}}\right)(s-t)} d s, \\
B(t) & =R_{m} e^{-\int_{t}^{T}(-\alpha(u)+\beta(u)+r) d u}+\int_{t}^{T} e^{-\int_{t}^{s}(-\alpha(u)+\beta(u)+r) d u} d s .
\end{aligned}
$$

The subsistence level case, i.e. $h(t)=h$, and the classical case, i.e. $h(t)=0$, can be easily obtained from the above results.

\section{Proof of proposition 2}

The value function is given by

$$
J(t, R(t)) \equiv \max _{\theta(t)} \mathbb{E}_{t}\left[\frac{\left(R(T)-R_{m}\right)^{1-\delta}}{1-\delta} e^{-\rho(T-t)}\right] .
$$

For this objective function, we can write the following HJB equation

$$
0=\max _{\theta(t)}\left\{\begin{array}{c}
-\rho J+\frac{\partial J}{\partial t}+\frac{\partial J}{\partial R}(R(t) r+\theta(t) S(t)(\mu-r)-c(t)) \\
+\frac{1}{2} \frac{\partial^{2} J}{\partial R^{2}} \theta(t)^{2} S(t)^{2} \sigma^{2}
\end{array}\right\},
$$

and the first order condition (FOC) of (35) w.r.t. $\theta(t)$ is

$$
\theta(t)^{*}=-\frac{\mu-r}{S(t) \sigma^{2}} \frac{\frac{\partial J}{\partial R}}{\frac{\partial^{2} J}{\partial R^{2}}}
$$

We assume the following guess function

$$
J(t, R(t))=A(t)^{\delta} \frac{(R(t)-B(t))^{1-\delta}}{1-\delta},
$$


where $A(t)$ is the function that must solve Eq. (35), with the boundary condition $A(T)=1$, while $B(t)$ must satisfy the boundary condition $B(T)=R_{m}$, and the optimal portfolio process $\theta(t)^{*}$ in (36), for our guess function can be written as

$$
\theta(t)^{*}=\frac{\mu-r}{S(t) \sigma^{2}} \frac{R(t)-B(t)}{\delta} .
$$

Substituting the optimal portfolio (38) and partial derivatives of the guess function into (35), we get

$$
0=\frac{\delta}{1-\delta} A_{t}+A(t)\left(r-y-\frac{\rho}{1-\delta}+\frac{1}{2} \frac{(\mu-r)^{2}}{\sigma^{2} \delta}\right)+\frac{A(t)}{R(t)-B(t)}\left(B(t)(r-y)-B_{t}\right),
$$

which can be separated into two differential equations, one that consists of the terms containing $(R(t)-B(t))^{-1}$ and one without them and after few simplifications, we have

$$
\left\{\begin{array}{l}
0=A_{t}+A(t)\left(\frac{1-\delta}{\delta}(r-y)+\frac{1-\delta}{2 \delta^{2}} \frac{(\mu-r)^{2}}{\sigma^{2}}-\frac{\rho}{\delta}\right), \\
0=B(t)(r-y)-B_{t} .
\end{array}\right.
$$

The above ordinary differential equations with their corresponding boundary conditions have the following solutions:

$$
A(t)=e^{-\left(\frac{\delta-1}{\delta} r-\frac{\delta-1}{\delta} y+\frac{\delta-1}{2 \delta^{2}} \frac{(\mu-r)^{2}}{\sigma^{2}}-\frac{\rho}{\delta}\right)(T-t)}, \quad B(t)=R_{m} e^{-(r-y)(T-t)} .
$$

\section{Proof of proposition 3}

The value function is given by

$$
J(t, R(t), c(t)) \equiv \max _{\theta(t)} \mathbb{E}_{t}\left[\frac{\left(R(T)-R_{m}\right)^{1-\delta}}{1-\delta} e^{-\rho(T-t)}\right] .
$$

For this objective function, we can write the following HJB equation

$$
0=\max _{\theta(t)}\left\{\begin{array}{c}
-\rho J+\frac{\partial J}{\partial t}+\frac{\partial J}{\partial R}(R(t) r+\theta(t) S(t)(\mu-r)-c(t)) \\
+\frac{\partial J}{\partial c}(1-\omega)(1+y)\left(\frac{y R(t)(1+r)+y \theta(t) S(t)(\mu-r)}{1+y}-c(t)\right) \\
+\frac{1}{2} \frac{\partial^{2} J}{\partial R^{2}} \theta(t)^{2} S(t)^{2} \sigma^{2}+\frac{1}{2} \frac{\partial^{2} J}{\partial c^{2}} a^{2} \theta(t)^{2} S(t)^{2} \sigma^{2}+\frac{\partial^{2} J}{\partial c \partial R} a \theta(t)^{2} S(t)^{2} \sigma^{2}
\end{array}\right\}
$$

and the first order condition (FOC) of (39) w.r.t. $\theta(t)$ is

$$
\theta(t)^{*}=-\frac{\mu-r}{S(t) \sigma^{2}} \frac{\frac{\partial J}{\partial R}+\frac{\partial J}{\partial c} a}{\frac{\partial^{2} J}{\partial R^{2}}+\frac{\partial^{2} J}{\partial c^{2}} a^{2}+2 \frac{\partial^{2} J}{\partial c \partial R} a} .
$$

We assume the following guess function 


$$
J(t, R(t), c(t))=A(t)^{\delta} \frac{\left(R(t)-B(t, c(t))^{1-\delta}\right.}{1-\delta} .
$$

Thus, the optimal portfolio process $\theta(t)^{*}$ in (40), can be written as

$$
\theta(t)^{*}=-\frac{\mu-r}{S(t) \sigma^{2}} A(t)^{\delta}(R(t)-B(t, c(t)))^{-\delta} \frac{1-B_{c} a}{F(t, c(t))},
$$

where we define

$$
\begin{aligned}
F(t, c(t))= & -\delta A(t)^{\delta}(R(t)-B(t, c(t)))^{-\delta-1}-\delta A(t)^{\delta}(R(t)-B(t, c(t)))^{-\delta-1} B_{c}^{2} a^{2} \\
& -A(t)^{\delta}(R(t)-B(t, c(t)))^{-\delta} B_{c c} a^{2}+2 \delta A(t)^{\delta}\left(R(t)-B(t, c(t))^{-\delta-1} B_{c} a .\right.
\end{aligned}
$$

The HJB Eq. (39), under the hypotheses $B_{c}=\eta$ and $B_{c c}=0$, becomes

$$
\begin{aligned}
0= & -A(t) \frac{\rho}{1-\delta}+\frac{\delta}{1-\delta} A_{t}-A(t) \frac{B_{t}}{R(t)-B(t, c(t)} \\
& +A(t) r+A(t) \frac{r B(t, c(t))}{R(t)-B(t, c(t))} \\
& -A(t) \frac{c(t)}{R(t)-B(t, c(t))}-A(t) \eta a(1+r)-A(t) \frac{\eta a(1+r) B(t, c(t))}{R(t)-B(t, c(t))} \\
& +A(t) \frac{\eta(1-\omega) c(t)}{R(t)-B(t, c(t))}+A(t) \frac{\eta a c(t)}{R(t)-B(t, c(t))}+\frac{A(t)(\mu-r)^{2}}{2 \sigma^{2} \delta},
\end{aligned}
$$

which can be separated into two differential equations, one that consists of the terms containing $(R(t)-B(t, c(t)))^{-1}$ and one without them and after few simplifications, we have

$$
\left\{\begin{array}{l}
0=A_{t}+A(t)\left(\frac{1-\delta}{\delta} r-\frac{\rho}{\delta}-\frac{1-\delta}{\delta} \eta a(1+r)+\frac{1-\delta}{\delta} \frac{(\mu-r)^{2}}{2 \sigma^{2} \delta}\right), \\
0=B_{t}-B(t, c(t))(r-\eta a(1+r))+c(t)(1-\eta(1-\omega)-\eta a),
\end{array}\right.
$$

and the optimal portfolio in this case can be written as using (40)

$$
\theta(t)^{*}=\frac{\mu-r}{\delta S(t) \sigma^{2}} \frac{R(t)-B(t, c(t))}{1-\eta a} .
$$

These equations are ordinary linear differential equations (ODEs) and their boundary conditions can be obtained from the boundary condition of the HJB Eq.: $A(T, c(T))=1$ and $B(T, c(T))=R_{m}$.

The solution of the ODE (42), together with their boundary conditions is given by

$$
A(t)=e^{-\left(\frac{\delta-1}{\delta} r+\frac{\rho}{\delta}-\frac{\delta-1}{\delta} \eta a(1+r)+\frac{\delta-1}{\delta} \frac{(\mu-r)^{2}}{2 \sigma^{2} \delta}\right)(T-t)} .
$$

Since the second differential equation has been obtained under the hypothesis that $B_{c}=\eta$ and $B_{c c}=0$, then the only consistent functional form for $B(t, c(t))$ is

$$
B(t, c(t))=\eta(t) c(t)+h(t),
$$

where $\eta(t)$ and $h(t)$ may be functions of time. Thus the second ODE can be rewritten as follows 


$$
\begin{aligned}
& \left(\frac{\partial \eta(t)}{\partial t} c(t)+\frac{\partial h(t)}{\partial t}\right) \\
& \quad-(r-\eta(t) a(1+r))(\eta(t) c(t)+h(t))-(\eta(t)(1-\omega+a)-1) c(t)=0,
\end{aligned}
$$

which can be separated into two ODEs, one which contains $c(t)$ and one which contains all the terms without $c(t)$

$$
\begin{gathered}
0=\frac{\partial \eta(t)}{\partial t}-(r-\eta(t) a(1+r)) \eta(t)-(\eta(t)(1-\omega+a)-1) \\
0=\frac{\partial h(t)}{\partial t}-(r-\eta(t) a(1+r)) h(t)
\end{gathered}
$$

with the boundary condition

$$
\eta(T) c(T)+h(T)=R_{m}
$$

as $B(T, c(T))=R_{m}$ and consequently $\eta(T)=0$ and $h(T)=R_{m}$, which means that $\eta$ must be a constant and the ODE (43) (with $\left.\frac{\partial \eta(t)}{\partial t}=0\right)$ gives

$$
\eta^{2} a(1+r)-\eta(1+r+a-\omega)+1=0,
$$

which has two solutions:

$$
\eta=\frac{(1+r+a-\omega) \pm \sqrt{(1+r+a-\omega)^{2}-4 a(1+r)}}{2 a(1+r)},
$$

where one of the two solutions $\left(\eta^{*}\right)$ must be chosen suitably.

The ODE (44), together with the boundary condition, has a unique solution

$$
h(t)=R_{m} e^{-\left(r-\eta^{*} a(1+r)\right)(T-t)} .
$$

Therefore,

$$
B(t, c(t))=\eta^{*} c(t)+R_{m} e^{-\left(r-\eta^{*} a(1+r)\right)(T-t)},
$$

such that $-\infty<\eta^{*}<\frac{1}{a}$.

\section{References}

Bajeux-Besnainou I, Ogunc K (2006) Spending rules for endowment funds: a dynamic model with subsistence levels. Rev Quant Financ Acc 27:93-107

Blume ME (2010) Endowment spending in volatile markets: what should fiduciaries do? Rev Quant Financ Acc 35(2):163-178

Cejnek G, Franz R, Randl O, Stoughton N (2014) A survey of university endowment management research. J Invest Manag Third Quart 40:40

Constantinides GM (1990) Habit formation: a resolution of the equity premium puzzle. J Political Econ 98(3):519-543

Dybvig PH (1999) Using asset allocation to protect spending. Financ Anal J 55(1):49-62

Ennis RM, Williamson JP (1976) Spending policy for educational endowments. Common Fund, Naugatuck, Connecticut

Fraser SP, Jennings WW (2010) Examining the use of investment policy statements. J Wealth Manag 13(2):10-22 
Gourinchas PO, Parker JA (2002) Consumption over the life cycle. Econometrica 70(1):47-89

Hansmann H (1990) Why do universities have endowments? J Leg Stud 19(1):3-42

Horneff V, Maurer R, Mitchell OS, Rogalla R (2015) Optimal life cycle portfolio choice with variable annuities offering liquidity and investment downside protection. Insur Math Econ 63((Supplement C)):91-107

Kaufman RT, Woglom G (2005) Modifying endowment spending rules: Is it the cure for overspending? J Educ Financ 31(2): 146-171

Merton RC (1969) Lifetime portfolio selection under uncertainty: the continuous-time case. Rev Econ Stat 51(3):247-257

Merton RC (1971) Optimum consumption and portfolio rules in a continuous-time model. J Econ Theory 3(4):373-413

Merton RC (1993) Optimal investment strategies for university endowment funds. Continuous-time finance. University of Chicago Press, Chicago

Munk C (2008) Portfolio and consumption choice with stochastic investment opportunities and habit formation in preferences. J Econ Dyn Control 32:3560-3589

NACUBO (2016) Nacubo-commonfound study of endowments (ncse). Technical report, National Association of College and University Business Officers

Samuelson PA (1969) Lifetime portfolio selection by dynamic stochastic programming. Rev Econ Stat 51(3):239-246

Sedlacek VO, Jarvis WF (2010) Endowment spending: building a stronger policy framework. Commonfund Institute, $\mathrm{p} 24$

Tobin J (1974) What is permanent endowment income? Am Econ Rev 64(2):427-432

Publisher's Note Springer Nature remains neutral with regard to jurisdictional claims in published maps and institutional affiliations. 